to support patients and to fast-track those with complex needs to clinic.

We describe views and experiences of people who chose Chlamydia-OCCP for treatment.

Methods An interviewer conducted 40 in-depth follow-up telephone interviews with a purposive sample. Thematic analysis. Results Participants described choosing Chlamydia-OCCP over alternatives for its expected greater speed, convenience and privacy; or trying it out without preconceptions. They valued rapid access to treatment, but also obtaining treatment when convenient.

Despite general concerns about keeping their chlamydia diagnoses secret, several accessed the online consultation immediately, at work/in public. Participants described Chlamydia-OCCP web-app as easy to use, with only minor technical or privacy issues. They found providing personal/sensitive information online acceptable, reasoning that: 1 . it was preferable to (potentially embarrassing) face-to-face consultations; 2 . ChlamydiaOCCP was associated with trusted services; 3 . they already provided personal information online for other services.

Barriers to rapid treatment via Chlamydia-OCCP included: difficulties accessing pharmacy due to employment/carer demands or holiday/business travel (these also impeded clinic access); pharmacy staff's knowledge of process. Collecting treatment from pharmacies was universally acceptable, despite difficulties experienced by some.

Reactions of participants fast-tracked to clinic varied (anxiety, disappointment, relief), and helpline contact reassured them, aiding their understanding of why a clinic visit was necessary.

Conclusion Participants expressed satisfaction with the web-app and helpline, using Chlamydia-OCCP to obtain treatment discreetly, conveniently and quickly, despite busy lifestyles. Further evaluation of the pharmacy process will explore how this can be optimised, to further improve time-to-treatment and satisfaction. Disclosure of interest statement Nothing to declare.

\section{P12.02 DEVELOPING AND USING THE ECLINICAL CARE PATHWAY FRAMEWORK: A NOVEL TOOL FOR CREATING ONLINE CLINICAL CARE PATHWAYS AND ITS APPLICATION TO MANAGEMENT OF GENITAL CHLAMYDIA}

1J Gibbs*, 'U Sutcliffe, ${ }^{2} V$ Gkatzidou, ${ }^{3} \mathrm{P}$ Sonnenberg, ${ }^{2} \mathrm{~K}$ Hone, ${ }^{1} \mathrm{R}$ Ashcroft, ${ }^{4} \mathrm{E}$ HardingEsch, ${ }^{4} \mathrm{C}$ Lowndes, ${ }^{5} \mathrm{ST}$ Sadiq, ${ }^{1} \mathrm{CS}$ Estcourt. 'Queen Mary University of London; ${ }^{2}$ Brunel University London; ${ }^{3}$ University College London; ${ }^{4}$ Public Health UK; ${ }^{5}$ St George's University of London

\subsection{6/sextrans-2015-052270.482}

Introduction Home sexually transmitted infection (STI) sampling and remote STI diagnostics create potential for online treatment. Currently there is no guidance on development or content of online clinical consultations and, particularly, no recommendations relevant to sexual health. We describe creation of a new framework for developing complex online clinical care pathways and its application to management of people with genital chlamydia.

Methods We developed the eClinical Care Pathway Framework (eCCPF) consisting of a nine-step iterative process, Step 1: determines aims of the online clinical care pathway; Step 2: defines the functional units of the pathway; Step 3: drafts the online clinical consultation; Step 4: expert review; Step 5: comprehension testing; Step 6: user-centred interface testing; Step 7: specification development; Step 8: usability testing and further comprehension testing; Step 9: pilots the pathway. We then applied the framework to create the Chlamydia Online Clinical Care Pathway (Chlamydia-OCCP).

Results Using the eCCPF enabled us to elucidate the different sequence of functional units of care, as in contrast to traditional medicine, the Chlamydia-OCCP starts with provision of test results (diagnosis). Users then obtain infection-related information, before completing an online automated clinical consultation (medical and sexual history, partner notification), before collection of antibiotics from a community pharmacy. This enables a more focussed approach to assessment of safety of antibiotic prescribing than lengthier traditional medication history questions.

Conclusion By following each step of the eCCPF, the resulting Chlamydia-OCCP has a different sequence to traditional care pathways and is adapted to the needs of remote testing and online care. It provides the clinical services and surveillance functions required to meet UK national standards. This standardised method of collecting data on demography and sexual behaviour, with easily extractable data and potential for interoperability with surveillance systems, could be a powerful tool for public health and clinical management.

Disclosure of interest statement Nothing to declare.

\section{P12.03 HOW ACCURATE AND COMPREHENSIVE ARE CURRENTLY AVAILABLE MOBILE MEDICAL APPLICATIONS (APPS) FOR SEXUALLY TRANSMITTED AND GENITAL INFECTIONS: A COMPREHENSIVE REVIEW}

'1 Gibbs*, ${ }^{2} V$ Gkatzidou, 'L Tickle, 'SR Manning, ${ }^{1} T$ Tilakkumar, ${ }^{2} \mathrm{~K}$ Hone, ${ }^{1}$ RE Ashcroft, ${ }^{3} \mathrm{P}$ Sonnenberg, ${ }^{4} \mathrm{ST}$ Sadiq, ${ }^{1} \mathrm{CS}$ Estcourt. 'Queen Mary University of London; ${ }^{2}$ Brunel University of London; ${ }^{3}$ University College London; ${ }^{4}$ St George's University of London

\subsection{6/sextrans-2015-052270.483}

Introduction In the UK, $88 \%$ of young people, a group at high risk of STIs, own a smartphone. Seeking sexual health advice online is common and provision of STI apps is increasing. The UK lacks a robust framework for quality control of mobile medical apps and potential for misinformation is substantial. We undertook a comprehensive review of content and accuracy of contemporary apps for STIs (except HIV) and related genital infections aimed at members of the public.

Methods Search of Googleplay and iTunes stores for free and paid apps regarding STIs and genital infections, testing, diagnosis and management, between 10/09/2014-16/09/2014. We assessed eligible apps against: 1) 19 modified Health on The Net (HON) Foundation principles; 2) comprehensiveness and accuracy of information on individual STIs/genital infections, and their diagnosis and management, compared with respective National Health Service STI information webpage content.

Results 144/6642 apps met eligibility criteria (Android $n=94$; iOS $n=29$; both $n=24)$. 57 were excluded after downloading. 87 apps (52 Android, 20 iOS, 15 both) were analysed. Only $29 \%$ apps met $>/=6 \mathrm{HON}$ criteria. Content was highly variable: $34 / 87$ (39\%) covered one or two infections; 16/34 (47\%) were ebooks, predominately about genital herpes or candidiasis; 40/87 (46\%) covered multiple STIs; $5 / 87$ (6\%), solely focused on accessing STI testing. In terms of accuracy: 13/87 (15\%) were fully, 46/87 (53\%) mostly and 28/87 (32\%) partially accurate. $25 / 87(29 \%)$ contained $>/=1$ instance of potentially harmful information. Apps available on both platforms had a greater degree of accuracy than single platform apps. Only 1 app 